\title{
Research on the Modeling and Transition Algorithm of a Novel Wall-Climbing Robot
}

\author{
Zhenhui $\mathrm{Li}^{1,2, a}$, Hongguang Wang ${ }^{1, b}$, Yuechao Wang ${ }^{1, \mathrm{c}}$, Aihua Liu ${ }^{1, \mathrm{~d}}$ \\ and Weiguang Dong ${ }^{1,2, \mathrm{e}}$ \\ ${ }^{1}$ State Key Laboratory of Robotics, Shenyang Institute of Automation, Chinese Academy of \\ Sciences, Shenyang 110016, China \\ ${ }^{2}$ Graduate School of the Chinese Academy of Sciences, Beijing 100039, China \\ alizhenhui@sia.cn, ’hgwang@sia.cn, cycwang@sia.cn, 'liuaihua@sia.cn \\ and ${ }^{\mathrm{e}}$ dongweiguang@sia.cn
}

Keywords: Wall-climbing Robot, Transition between inclined surfaces, FSM

\begin{abstract}
This paper presents a modeling and transition algorithm of a novel wall-climbing robot with biped-wheel hybrid mechanism. In order to realize robot transitions between inclined surfaces, the robot's locomotion gait is analyzed and a locomotion gait planning model based on Finite State Machine (FSM) is established. Moreover a transition algorithm between inclined surfaces is proposed based on multi-sensors data fusions and logical reasoning networks. The results of simulations and experiments show that the model and algorithm are valid and can be applied for the wall-climbing robot's transition between the concave surfaces.
\end{abstract}

\section{Introduction}

With the development of robot technology, a number of applications of wall-climbing robots have been found in military and industrial fields. According to the locomotion ability, wall-climbing robots can be classified into wheel-type, pedrail-type and foot-type. The wheel and pedrail wall-climbing robots such as City-climber [1] and Alica [2] have a good ability in movement, but they can only work on even walls and can not transfer between inclined surfaces. Foot-type wall-climbing robots such as Robug II [3] and Crawler [4] have varied structures and a good ability in obstacle negotiation, but they can not move fast.

The novel biped-wheel wall-climbing robot combines the abilities in movement of wheel robots with obstacle negotiation of foot robots. It can move fast on even walls and transfer between inclined surfaces. The transition means the robot moves from a wall to another that is crossed with the former. However, the efficiency and accuracy of the wall-climbing robot can not achieve the optimization if the robot is manually manipulated by human operators in transition. In order to achieve an autonomous transition between inclined surfaces, Alsalam etc [5], J. Z. Xiao etc [6], Y. Jiang etc [7][8] have researched respectively the autonomous methods of transition between inclined surfaces for four-footed and biped wall-climbing robots. For the novel mechanism of the biped-wheel wall-climbing robot based on planetary gearing, there are no appropriate autonomous transition algorithms between inclined surfaces yet.

This paper focuses on the transition algorithm of a novel biped-wheel wall-climbing robot with biped-wheel hybrid mechanism. In order to realize robot transitions between inclined surfaces, the robot's locomotion gait is analyzed and a locomotion gait planning model based on Finite State Machine (FSM) is established. Moreover a transition algorithm between inclined surfaces is proposed based on multi-sensors data fusions and logical reasoning networks.

\section{Description of the Wall-climbing Robot}

As shown in Fig.1 (a), the wall-climbing robot consists of linkage, aerodynamic attraction module and vacuum suction module. The semicircular surfaces of the two modules structure an external meshed gearing and a linkage joins their centers, which form a planetary gearing system. As shown in 
Fig. 1 (b), the robot has 4 degrees freedom, including 3 rotary joints and 1 joint. Rotary joints $J_{1}$ and $J_{2}$ provide the robot movement and turning capability. Rotary joint $J_{3}$ allows the two modules rolling. Joint $J_{4}$ allows the robot extended and draws back its leg so that the suction cup can adsorb the surface firmly to accomplish transition between inclined surfaces.

The novel wall-climbing robot needs a self-contained embedded control system, thus minimization of the weight and power consumption is critical. The TMS320F28335 digital signal processor (DSP) from Texas Instruments Inc. (TI) is used as an embedded controller of the robot's control system. The driver of DC servo motors uses SN754410 from TI. Every DC motor has an encoder in order to control the position and velocity. The novel wall-climbing robot has a multi-sensors system that consists of encoders, voltage sensors, current sensors, pressure sensors, touch sensors, infrared sensors and proximity sensors. The diagram of hardware components of the robot control system are showed in Fig.2.

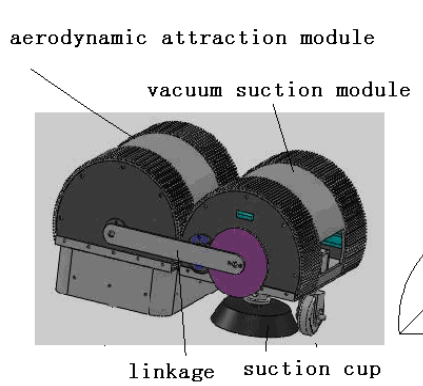

(a)

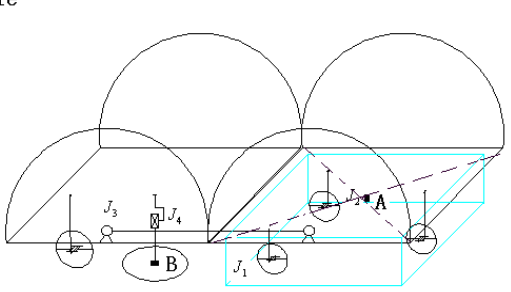

(b)

Fig. 1. A Wall-climbing robot

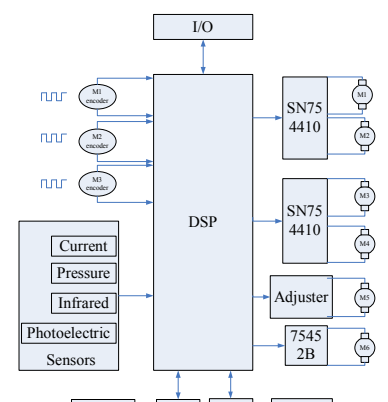

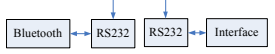

Fig. 2. Hardware components of the robot control system

\section{Gait Modeling and Transition Algorithm}

Locomotion Gait Formation. The novel wall-climbing robot accomplishes the transition between inclined surfaces by the cooperation of the aerodynamic attraction module, the vacuum suction module and the planetary gearing structure. When the robot moves from a surface to another with the angle $\beta(\beta \in[\pi / 2, \pi])$, the locomotion gait formation is shown in Fig.3.
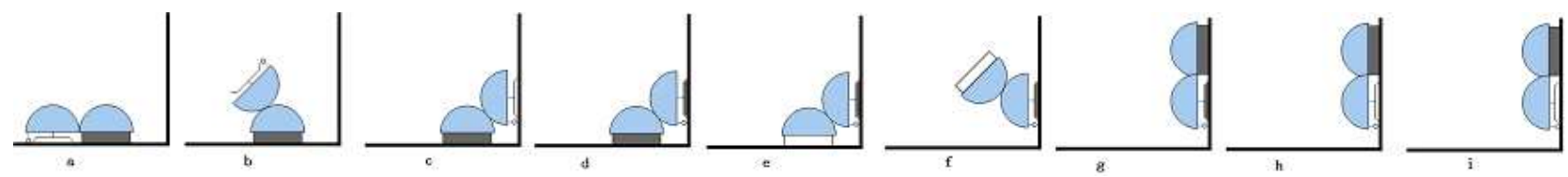

Fig. 3. The locomotion gait formation

The transition gait is described as follows:

The function of the locomotion gait of the transition between inclined surfaces for the robot can be expressed as Eq.1.

$$
G_{\beta}=g\left(L, \theta_{1}, \theta_{2}, \theta_{3}, l\right)
$$

where $\beta$ is the angle between inclined surfaces, and $L, \theta_{1}, \theta_{2}, \theta_{3}, l$ are the parameters of the function.

Locomotion Gait Modeling Based on FSM. Finite State Machine (FSM) consists of the finite states and the transfers between them. The robot can only be in one of the states. When the robot receives an input, FSM will produce an output, and an action or transition may happen.

The locomotion gait modeling based on FSM of the robot can be expressed as Eq.2.

$$
M=\left(Q, \Omega, \delta, q_{0}, F\right)
$$


where $Q$ is the set of finite states of the robot gait, $q_{i}$ is one state of $Q$. $\Omega$ is the set of inputs of the robot. $q_{0} \in Q$ is the original state of the robot. $F$ is the final state of the robot. The function of state action is $\delta: Q \times \Omega \rightarrow Q$, then $\delta\left(q_{i}, a\right)=q_{j}$ means that the robot transfers from the state $q_{i}$ to $q_{j}$ when the input is $a(a \in \Omega)$. The elements of the FSM of the novel robot take up 2 bytes, as shown in Table 1.

Table 1 The elements of the FSM of the novel robot

\begin{tabular}{cccccccccc}
\hline Bit & $12-15$ & $9-11$ & $7-8$ & $5-6$ & 4 & 3 & 2 & 1 & 0 \\
\hline Element & 0000 & LM & J3 & J4 & SC & AA & AR1 & AR2 & HS \\
\hline
\end{tabular}

LM (locomotion mode): " 000 "denotes the stop state of the robot. " 001 "denotes the go-forward state of the robot. " 010 " denotes the go-back state of the robot. " 011 " denotes the turn-left state of the robot. " $100 "$ denotes the turn-right state of the robot. " 101 " denotes the transition state of the robot. "110"and "111" denote the unlawful states.

J3: " 00 "denotes the stop state of the joint $J_{3}$. "01" denotes the clockwise rotation state of the joint $J_{3}$. " 10 " denotes the counter-clockwise rotation state of the join $J_{3}$. " 11 "denotes the adjusting state.

J4: " 00 "denotes the stop state of the joint $J_{4}$. " 01 " denotes the clockwise rotation state of the joint $J_{4}$. " 10 " denotes the counter-clockwise rotation state of the join $J_{4}$. " 11 "denotes the adjusting state.

SC(suction cup): " 0 "denotes the release state of the suction cup. " 1 "denotes the grip state of the suction cup.

AA (aerodynamic attraction): "0"denotes the stop state of the centrifugal motor. "1"denotes the start state of the centrifugal motor.

AR1 (adaptive regulate): " 0 " denotes the stop state of the pose adjusting for the aerodynamic attraction cup. " 1 " denotes the start state of the pose adjusting for the aerodynamic attraction cup.

AR2 (adaptive regulate): " 0 " denotes the stop state of the pose adjusting for the suction cup. " 1 " denotes the start state of the pose adjusting for the suction cup.

HS (handshake stage): " 0 "denotes that the robot is in the handshake stage. " 1 "denotes that the robot is out off the handshake stage.

According to the definition of the state vector of FSM for the robot locomotion gait, the state action route of the robot transition is shown in Fig. 4. $a_{i} \in \Omega$ is the input of the FSM, and it is also the guard of state action.

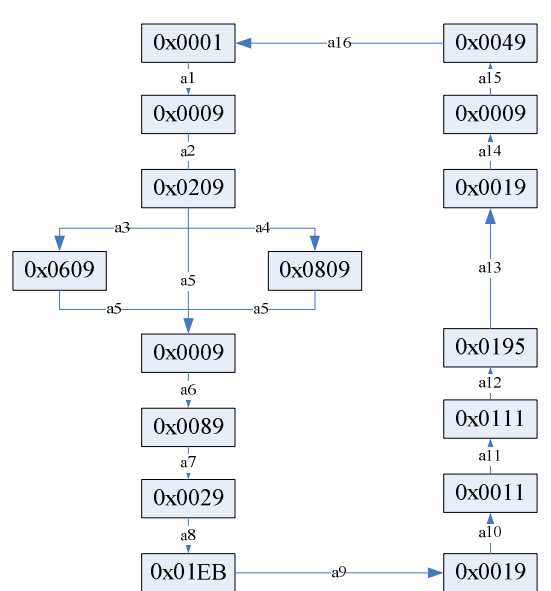

Fig. 4. The state action route of the transition

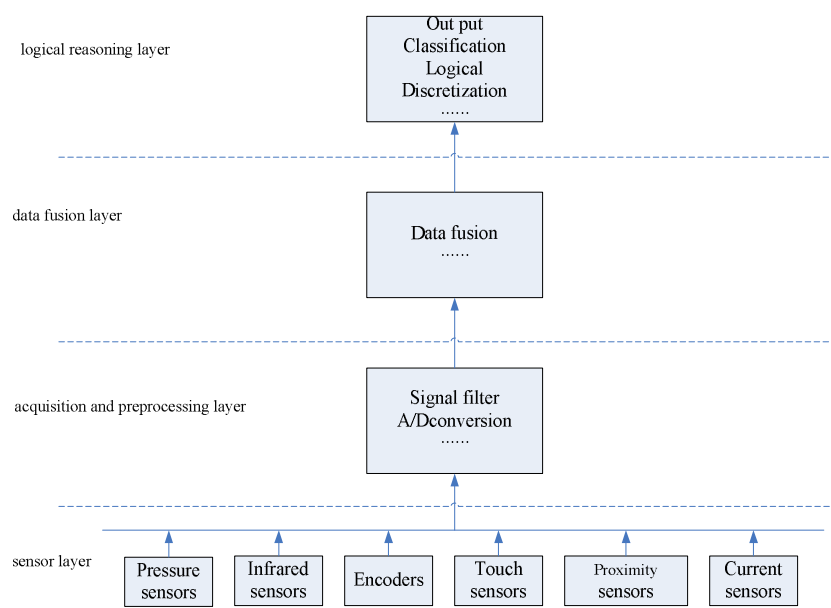

Fig. 5. A model for the multi-sensor data fusion process 
Transition Algorithm Based on Multi-sensor Data Fusion. Single sensor can only acquire unilateral environmental information. Multi-sensor data fusion acquires a synergistic combination of information gathered by various sensors to provide a better understanding of the environment. It refers to the acquisition, processing, fusion, and output of the information from sensors.

The novel wall-climbing robot has a multi-sensor system that consists of encoders, voltage sensors, current sensors, pressure sensors, touch sensors, infrared sensors, and proximity sensors. The wall-climbing robot understands the information of exterior and self states by the multiple sensors. A model for the multi-sensor data fusion process of the wall-climbing robot includes four layers as shown in fig.5. They are sensor layer, acquisition and processing layer, data fusion layer and logical reasoning layer.

1) Sensor layer: The pressure sensors monitor the pressure level inside the suction cup and aerodynamic attraction cup. The touch sensors provide the touch information of the suction cup. The infrared sensors under the vacuum suction module are used to measure the distance and parallelism between the suction cup and the target surface. The proximity sensors on the head of the robot are used to measure the distance between the robot and the barriers. The encoders and current sensors are responsible for the movement of each motor.

2) Acquisition and preprocessing layer: The Acquisition and preprocessing of the information from sensors includes A/D conversion and signal filter.

3) Data fusion layer: The data fusion layer is the core of the model. It completes some fusion computing, such as comparing, estimating, classification, etc, based on some special fusion rules.

\section{Examples:}

a) The robot has four infrared sensors under the vacuum suction model that are used to measure the distance and parallelism between the suction cup and the target surface. Let $L_{1}$ denote the distance and $H_{1}$ denote the parallelism. Two proximity sensors on the head of the robot are used to measure the distance and parallelism between the robot and the barriers. Let $L_{2}$ denote the distance and $H_{2}$ denote the parallelism. Thus, we have

$$
\begin{aligned}
& L_{1}=\sum \frac{l_{i}}{4}, i=1,2,3,4 \\
& H_{1}=\sqrt{\frac{\left(l_{1}-l_{3}\right)^{2}+\left(l_{2}-l_{4}\right)^{2}}{2}} \\
& L_{2}=\sum \frac{l_{i}}{2}, i=5,6 \\
& H_{2}=\left|l_{5}-l_{6}\right|
\end{aligned}
$$

where $l_{i}$ represents the distance from infrared sensor $i$ to the target surface, $i=1 \ldots 6$.

b) The robot has four touch sensors on the suction cup. Let $P_{1}$ denote the probability of the contact between the foot and the target surface, i.e.

$$
P_{1}=\frac{\sum T_{i}}{4} \times 100 \%, i=1,2,3,4
$$

where $T_{i}$ represents the state of the touch sensor $i, i=1,2,3,4$.

$T_{i}=\left\{\begin{array}{c}0 \text { if sensor } i \text { no touches } \\ 1 \text { if sensor } i \text { touches }\end{array}\right.$

c) The robot has four motors. Let $P_{2}$ denote the probability of motor locked rotor, i.e.

$$
P_{2}=\frac{\sum I_{i}}{4} \times 100 \%, \quad i=1,2,3,4
$$


where $I_{i}$ represents the state data of the current sensor $i, i=1,2,3,4$.

$$
I_{i}= \begin{cases}0 & \text { if current sensor } i<I \\ 1 & \text { if current sensor } i \geq I\end{cases}
$$

4) Logical reasoning layer: The multi-sensor data fusion aims at providing a better understanding of the environment to help the robot acquire the guards of state transfer. The outputs of data fusion layer are the inputs of logical reasoning layer that are the guards of state transfer $\left(a_{i} \in \Omega\right)$ of FSM. The logical reasoning layer consists of inputs, fuzzification, reasoning, combination, and outputs, as shown in Fig.6.

a) Input: The inputs come from the results of data fusion, including $P_{2}, P_{1}, H_{1}, L_{1}, H_{2}, L_{2}$, $P P_{1}$ (the pressure inside aerodynamic attraction cup ), $P P_{2}$ (the pressure inside the suction cup ).

b) Fuzzification: $P_{2}$ and $P_{1}$ are dispersed into 1, 0 and $0.5(1>P>0) . H_{1}, L_{1}, H_{2}, L_{2}, P P_{1}$ and $P P_{2}$ are dispersed into 1 and 0 .

c) Reasoning: The reasoning is regarded as a multi-input-multi-output (MIMO) system. A MIMO system can always be separated into a group of multi-input-single-output (MISO) system. A MISO system is a reasoning based on IF-THEN rules. The $m$-th rule is in the following form:

If $x_{1}$ is $x_{1}^{m}, \ldots$ and $x_{i}$ is $x_{i}^{m}$, then $\mathrm{y}$ is $\mathrm{B}$, with $\mathrm{m}=1 \ldots \mathrm{n}$, where $x_{i}$ is the input variables and $\mathrm{B}$ is the 3 rd layer variables of the networks, $x_{i}^{m} \in\{0,0.5,1\}$.

d) Combination and output: Combine the results of reasoning. The output $y_{n}$ is the guard of state action $a_{i} \quad\left(a_{i} \in \Omega\right)$ of FSM.

\section{Simulation and Experiment}

The size of the robot for simulation and experiment is $\mathrm{R}=10 \mathrm{~cm}$ (radius of planetary gearing), $l=3 \mathrm{~cm}$ (leg of suction cup), and $a=2 \mathrm{~cm}$ (distance from leg to the center of vacuum suction module). A is appointed as reference point of aerodynamic attraction module, and B is appointed as reference point of vacuum suction module (shown as Fig.2). When $\beta \in[\pi / 2, \pi]$, the relationship between $\mathrm{L}$ (the optimal distance from the start of the robot to the target surface) and $\beta$ is:

$$
L=\frac{R}{\sin \left(\frac{\beta}{2}\right)}+\frac{l}{\sin (\pi-\beta)}-R
$$

when the robot transfers between inclined surfaces, neither $\beta$ nor $\mathrm{L}$ is known. $\mathrm{L}=5 \mathrm{~cm}$ (the optimal distance when $\beta=2 \pi / 3$ ) is appointed as a parameter of transition algorithm.

Fig. 7 shows the trajectory of $\mathrm{A}$ and $\mathrm{B}$ when the robot transfers between inclined surfaces when $\beta=2 \pi / 3$. Fig. 8 shows the trajectory of $\mathrm{A}$ and $\mathrm{B}$ when $\beta=\pi / 2$. Fig.9 shows the trajectory of $\mathrm{A}$ and $\mathrm{B}$ when $\beta=3 \pi / 4 . \gamma$ is the angle between suction cup and the target surface. The simulation results show that the transition algorithm can accomplish a concave transition fast and reliably, and that the transition algorithm has a good astringency.

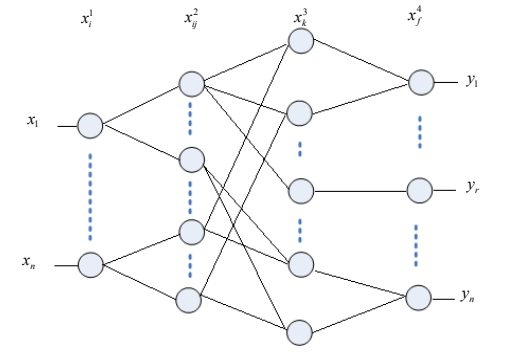

Fig. 6. The structure of logical reasoning layer

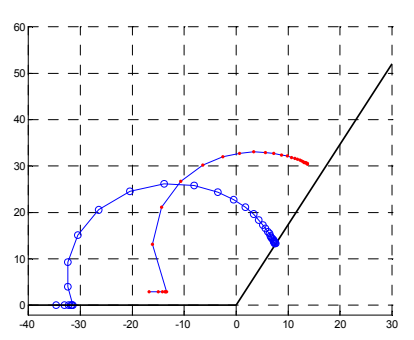

Fig. 7. The trajectory when $\beta=2 \pi / 3$ 


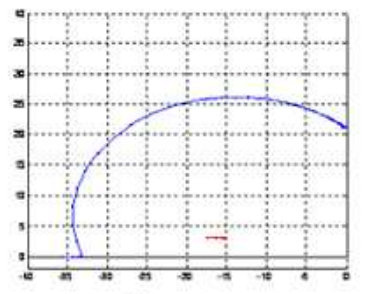

a

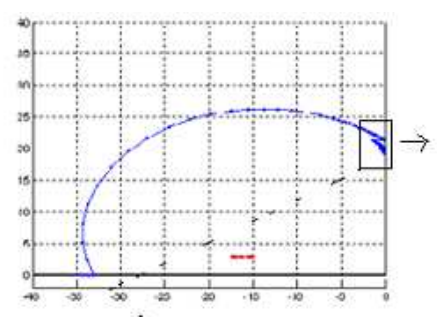

b

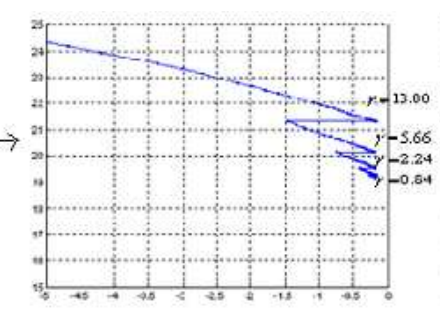

c

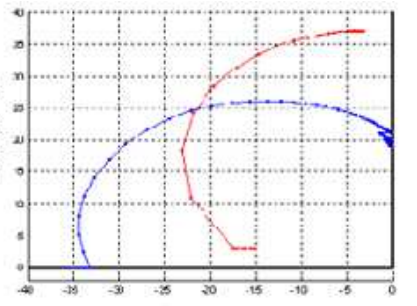

d

Fig. 8 . The trajectory when $\beta=\pi / 2$
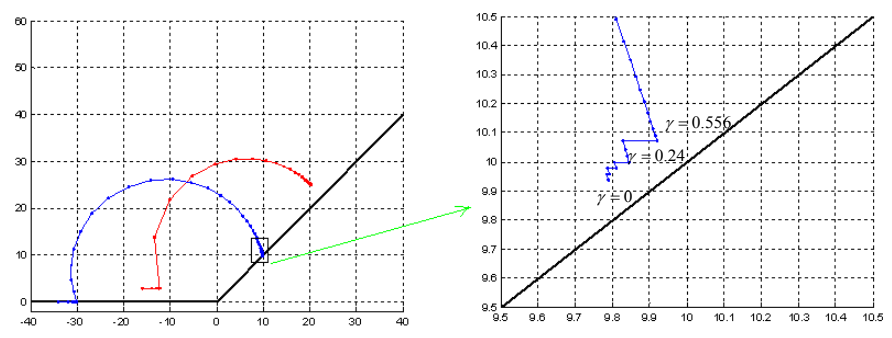

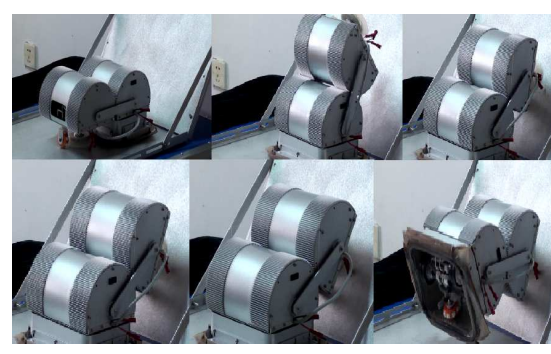

Fig. 9. The trajectory when $\beta=3 \pi / 4$

Fig. 10. The transition experiment of the robot

The transition experiment of the robot is shown as Fig.10. The experiment is conducted on a concave surface with $\beta=2 \pi / 3$ built with 2 pieces of aluminous boards. The results of the experiment show that the algorithm can successfully help the wall-climbing robot complete a concave transition automatically with $\beta$ unknown.

\section{Conclusion}

This paper is motivated by the problem of automated concave transition of wall-climbing robots. A locomotion gait planning model based on FSM is established. A transition algorithm between inclined surfaces based on multi-sensors data fusions and logical reasoning networks is proposed. The results of simulations and experiments prove that the model and the proposed algorithm are valid.

\section{References}

[1] M. Eliott, W. Morris, A. Calle, J. Z. Xiao, City-Climbers at Work,J. 2007 IEEE International Conference on Robotics and Automation. April 10-14, 2007, Roma, Italy.2007IEEE, 2764 2765.

[2] Longo. D, Muscato. G, The Alicia climbing robot: A three-model robot for automatic wall inspection, J. IEEE Robotics and Automation Magazine, 13(2006), 42-50.

[3] Luk.B.L, Collie.A.A, Billingsley.J, Robug II : An intelligent wall climbing robot, J. Proceedings of IEEE International Conference on Robotics and Automation, Piscataway, NJ, USA: IEEE, 1991. 2442-2347.

[4] Xiao. J. Z, Minor. M, Dukimarta. H, Modeling and control of an under-actuated miniature crawler robot, J. Proceedings of the IEEE/RSJ International Conference on Robotics and Systems, Piscataway, NJ, USA: IEEE, 2001. 1546-1551.

[5] Alsalameh.A.A.S, Amin.S.H.M, Mamat.R, Mechanical design of a quadruped robot for horizontal ground to vertical wall movement. Proceedings TENCON 2000, 2000.

[6] J.Z.Xiao, N. Xi, J.Xiao, J.D.Tan, Multi-sensor referenced gait control of a miniature climbing robot. IEEE/RSJ International Conference on Robotics and Systems, 2003

[7] H.G.Wang, Y.Jiang, L.J.Fang, M.Y.Zhao, Gait planning of concave transition between different slopes for bipedal wall-climbing robots, J. CAAI Transactions on Intelligent Systems, 4(2007) 40-45

[8] Y.Jiang, H.G.Wang, L.J.Fang, M.Y.Zhao, A novel approach to fault detection and identification in suction foot control of a climbing robot, IEEE/RSJ International Conference on Robotics and Systems, October 9-15,2006, Beijing, China. 2006IEEE, 3422 3428. 